\title{
The Influence of Reactive Oxygen Species on "Respiration" Isotope Effects
}

\author{
KEVIN M SUTHERLAND, JORDON D HEMINGWAY AND \\ DAVID T JOHNSTON \\ Harvard University \\ Presenting Author: ksutherland@fas.harvard.edu
}

The biogeochemical fluxes that produce and consume $\mathrm{O}_{2}$ play a critical role in regulating Earth's climate and habitability. The triple oxygen isotope method is one of the most robust tools for directly tracing oxygen cycling and measuring gross primary productivity in the marine environment. This method assumes that photosynthesis, microbial oxygen consumption (in the form of respiration), and gas exchange are the primary influences on dissolved oxygen, and that they have predictable, consistent isotopic end-members. Recent work, however, has highlighted the ubiquitous production of extracellular reactive oxygen species (e.g., superoxide and hydrogen peroxide) by microorganisms. This flux may be responsible for as much as 20 percent of net oxygen utilization in the ocean. Such prolific production of ROS commands that enzymes and chemical reactions involved in ROS production and degradation be considered as both source and sink in marine triple oxygen isotope mass balance. In this study, we measure the triple oxygen isotope effects of superoxide dismutase, catalase, and ironmediated $\mathrm{H}_{2} \mathrm{O}_{2}$ degradation to examine the influence ROSmediated recycling of $\mathrm{O}_{2}$ on oxygen utilization isotope effects. We incorporate these analytical constraints into an oxygen isotope flux model to determine the potential role of ROSmediated oxygen recycling in explaining wide disparities in "respiration" isotope effects in previous field and lab studies. We find that ROS-mediated oxygen cycling can reconcile the range of triple oxygen isotope fractionation factors presented in previous studies, and that typical marine isotope effects are largely consistent with estimates of superoxide-mediated oxygen loss. Lastly, we highlight the apparent likelihood of noncanonical triple oxygen isotope mass-laws around 0.500 in ROS production. Altogether, we make the case that a more comprehensive consideration of ROS-mediated recycling of $\mathrm{O}_{2}$ is needed to improve oxygen isotope-based estimates of gross primary productivity and our overall understanding of electron flows in the global ocean. 